\title{
Repeatability of the Measurement of Exhaled Volatile Metabolites Using Selected Ion Flow Tube Mass Spectrometry
}

\author{
Piers R. Boshier, ${ }^{a}$ Nandor Marczin, ${ }^{b}$ and George B. Hanna ${ }^{a}$ \\ ${ }^{a}$ Department of Biosurgery and Surgical Technology, Imperial College London, St. Mary's Hospital, London, \\ United Kingdom \\ ${ }^{\mathrm{b}}$ Department of Anaesthetics, Pain Medicine, and Intensive Care, Imperial College London, Chelsea and \\ Westminster Hospital, London, United Kingdom
}

Selected ion flow tube mass spectrometry, SIFT-MS, has been used to determine the repeatability of the analysis of volatile metabolites within the breath of healthy volunteers, with emphasis on the influence of sampling methodology. Baseline instrument specific coefficients of variability for examined metabolites were as follows: acetone $(1 \%)$, ammonia ( $1 \%)$, isoprene $(2 \%)$, propanol $(6 \%)$, ethanol $(7 \%)$, acetic acid $(7 \%)$, and hydrogen cyanide $(19 \%)$. Metabolite concentration and related product ion count rate were identified as strong determinants of measurement variation. With the exception of ammonia, an orally released metabolite, variability in repeated on-line breath analysis tended to be lower for metabolites of systemic origin. Standardization of sampling technique improved the repeatability of the analysis of selected metabolites. Off-line (bag) alveolar breath sampling, as opposed to mixed (whole) breath sampling, likewise improved the repeatability of the analysis of all metabolites investigated, with the exception of acetic acid. We conclude that SIFT-MS analysis of common volatile metabolites within the breath of healthy volunteers is both reliable and repeatable. For selected metabolites, the finding that repeatability is improved through modification of sampling methodology may have implications in terms of future recommended practices. (J Am Soc Mass Spectrom 2010, 21, 1070-1074) (c) 2010 American Society for Mass Spectrometry

$\mathrm{T}$ The analysis of volatile metabolites within exhaled breath is a promising field of medical research offering a safe and noninvasive approach to disease diagnosis and therapeutic monitoring. In spite of those potential advantages, the invasive collection and analysis of human body fluids and tissues remains the cornerstone in modern medical practice. Historic difficulties associated with accurate and reliable detection of volatile metabolites at levels characteristic of exhaled breath, typically equivalent to parts-per-million (ppm) and parts-per-billion ( $\mathrm{ppb}$ ), are partly responsible for the limited success of clinical breath testing. Recent advances have, however, overcome these issues and have led to the development of techniques such as selected ion flow tube mass spectrometry, SIFT-MS, which combines quantitative mass spectrometry and fast flow tube techniques [1]. By this approach, on-line and real time detection of a range of metabolites within a single exhalation can be achieved. Nevertheless, there remains an important requirement to determine the degree of instrument-specific variability in measuring different exhaled metabolites. Likewise, the influence of

Address reprint requests to Professor G. B. Hanna, Department of Biosurgery and Surgical Technology, Imperial College London, 10th Floor QEQM Wing, St. Mary's Hospital, London W2 1NY, UK. E-mail: g.hanna@ imperial.ac.uk. methodological variability must also be considered. Such information is essential as it will help support both appropriate selection of sampling methodology and interpretation of whether an observed difference in repeated measurements is likely to represent a true change in clinical status.

In this communication, we investigate the repeatability of the measurement of prominent exhaled metabolites in healthy subjects using SIFT-MS.

\section{Methods}

\section{Breath Sampling Methodology}

Breath samples were analyzed using the multiple ion monitoring (MIM) mode of the Profile-3 SIFT-MS instrument (Instrument Science, Crewe, UK), a full description of which is provided elsewhere [1]. Breath samples were collected by asking subjects to inhale deeply, close to total lung capacity, and to then promptly provide a complete exhalation into the sampling device. Calculation of metabolite concentration within on-line breath samples was performed by integration over the duration of the expiratory plateau. Alternatively, calculation of metabolite concentrations within off-line (bag) samples was performed by deter- 
mining their levels over a 60 s MIM mode scan. Off-line samples were left for a period of $1 \mathrm{~h}$ before analysis, reflecting the average duration between sampling and analysis commonly observed within the clinical setting. With the exception of water content, breath samples are stable during this period [2]. To eliminate condensation, all sample lines were heated and off-line samples were placed within an incubator held at $37^{\circ} \mathrm{C}$ both $5 \mathrm{~min}$ before and for the duration of the analysis.

Breath metabolites investigated in the present study were as follows: acetone, ethanol, propanol, ammonia, hydrogen cyanide, isoprene, and acetic acid. The quantification of these metabolites by SIFT-MS has been previously described [3, 4].

Analysis of repeatability was performed on logtransformed data through computation of Lin's concordance correlation coefficient [5] and the mean intraindividual coefficient of variability, determined as the average ratio of the standard deviation and the mean of paired datasets. Use of log-transformed data is in accordance with previous studies which show the concentrations of common breath metabolites follow more closely a log-normal distribution [6].

\section{Experiment I: Instrument-Specific Variability}

Healthy subjects $(n=10)$ were asked to provide single mixed (whole) breath samples by exhaling directly into single-use sample bags $(\sim 2 \mathrm{~L})$ constructed from double thickness $(2 \times 25 \mu \mathrm{m})$ Nalophan (Kalle UK Ltd., Witham, UK). Metabolites within collected samples were analyzed, and immediately $(<10 \mathrm{~s})$ re-analyzed to assess instrument specific variability. Changes in sample metabolite composition between repeat analyses were considered to be negligible.

\section{Experiment II: On-Line Control of Expiratory Pressure and Flow}

Healthy subjects $(n=10)$ were requested to exhale into the entry port of the SIFT-MS instrument. Volatile metabolites were analyzed within three repeat breaths. This process was repeated using a standardized protocol, for which the entry port of the SIFT-MS instrument was integrated with an independent LR2500 multiplegas analyzer (Logan Research Ltd., Rochester, UK), the latter allowing expiratory flow rate and mouth pressure to be set at $50 \mathrm{~mL} / \mathrm{s}$ and $>5 \mathrm{~cm} \mathrm{H}_{2} \mathrm{O}$, respectively (Figure S1 available as supplementary material, which can be found in the electronic version of this article).

\section{Experiment III: Off-Line Sampling from Different Respiratory Tract Compartments}

The repeatability of two off-line breath sampling methodologies was assessed. Mixed off-line breath samples $(n=2)$ were collected in short succession from 10 healthy volunteers. Alveolar breath samples $(n=2)$ were collected from 10 healthy volunteers using a $\mathrm{CO}_{2}$ triggered 'alveolar' breath sampler (developed at Innsbruck Medical University, Innsbruck, Austria), within which Nalophan sample bags were placed (Figure S2 available as supplementary material). At the preselected activation threshold, $4 \% \mathrm{CO}_{2}$, an average of three exhalations were required to collect a sample of $\sim 2 \mathrm{~L}$.

\section{Results and Discussion}

\section{Subjects}

Sixteen healthy, non-smoking subjects (4 female and 12 male; $29 \pm 7$ y) were enrolled to take part in one or more of the proposed studies. Testing by one-way ANOVA showed no significant difference in the mean age, height, weight, body mass index (BMI), or time since last oral intake between subjects participating in different experiments.

\section{Experiment I}

The repeatability of SIFT-MS was examined through the immediate re-analysis of mixed breath samples. Excellent repeatability, as indicated by a Lin's concordance coefficient, Rc, $>0.9$ [5] was observed for the major breath metabolites acetone, isoprene and ammonia (Table 1). For all metabolites examined observed mean coefficients of variability $(\mathrm{Cv})$, were $<20 \%$. Plotting Cv as a function of total product ion count rate and concentration (Figure 1) established signal intensity as a key determinant of measurement variation. In addition, the predicted measurement error, determined by the instrument software from the total number of counts recorded [7], matched approximately the observed difference (bias) between repeated analyses' of the same sample. It was also noted that the repeat analysis of a sample tended to yield higher metabolite concentrations, as indicated by a negative bias. As only a few samples were investigated, the significance of this finding remains uncertain. It may, however, be the case that there is an initial loss of sample onto the clean surfaces of the instrument. Further investigation of instrumentspecific variability using accurately known gas standards is therefore recommended. As intra-day and inter-day repeatability of the instrument were not investigated, further work is also required in this regard.

\section{Experiment II}

To investigate the influence of standardization of expiratory pressure and flow, a custom manifold was developed to allow control of these parameters. Median values and repeatability statistics are presented in Table 2. Results suggest that standardization can improve re- 
Table 1. Instrument specific variability

\begin{tabular}{|c|c|c|c|c|c|c|c|c|c|}
\hline & \multirow[b]{2}{*}{ Precursor ions } & \multirow[b]{2}{*}{ Product ion(s) } & \multicolumn{2}{|c|}{ Median } & \multirow{2}{*}{$\frac{\text { Predicted error }^{\mathrm{b}}}{( \pm \mathrm{ppb})}$} & \multirow{2}{*}{$\frac{\mathrm{Bias}^{\mathrm{c}}}{(\mathrm{ppb})}$} & \multirow{2}{*}{$\frac{\text { Precision }^{d}}{(p p b)}$} & \multirow[b]{2}{*}{$\mathrm{Rc}^{\mathrm{e}}$} & \multirow[b]{2}{*}{$\mathrm{Cv}^{f}$} \\
\hline & & & $\mathrm{ppb}$ & Ion count ${ }^{a}$ & & & & & \\
\hline Acetone & {$[\mathrm{H} 3 \mathrm{O}+]$} & 59,77 & 525 & 478 & 21 & -16 & 52 & 0.99 & 0.01 \\
\hline Ethanol & {$[\mathrm{H} 3 \mathrm{O}+]$} & 83 & 186 & 12 & 56 & -53 & 124 & 0.52 & 0.07 \\
\hline Propanol & {$[\mathrm{H} 3 \mathrm{O}+]$} & 43 & 23 & 16 & 4 & 3 & 8 & 0.70 & 0.06 \\
\hline Hydrogen cyanide & {$[\mathrm{H} 3 \mathrm{O}+]$} & 28 & 9 & 11 & 3 & -2 & 5 & 0.39 & 0.19 \\
\hline Isoprene & {$[\mathrm{NO}+]$} & 66,68 & 184 & 51 & 31 & -8 & 30 & 0.94 & 0.02 \\
\hline Acetic acid & {$[\mathrm{NO}+]$} & 90 & 107 & 14 & 29 & -25 & 98 & 0.37 & 0.07 \\
\hline Ammonia & {$[02+]$} & 17,35 & 1192 & 310 & 85 & -44 & 311 & 0.98 & 0.01 \\
\hline
\end{tabular}

aTotal number of product ion counts registered during a $60 \mathrm{~s}$ MIM mode scan.

${ }^{b}$ Average predicted measurement error determined from the total number of counts (generated by SIFT-MS software, see Reference [7]).

${ }^{\mathrm{c} B i a s,}$ mean difference between repeated measures.

dPrecision, standard deviation of the differences between repeated measures.

${ }^{e} \mathrm{Rc}=$ Lin's concordance correlation coefficient.

${ }^{\mathrm{f}} \mathrm{C} \mathrm{v}=$ mean coefficient of variability.

peatability of the analysis of acetone, propanol, hydrogen cyanide, and isoprene. Although these findings may reflect a reduction in the influence of methodological sources of variability; the precise mechanisms whereby such effects are mediated remain uncertain.

The site of release of breath metabolites within the airways and its relation to the chosen sampling methodology may contribute to observed variability. Several recent publications comparing metabolite levels in mouth- and nose-exhaled breath and within the oral cavity, would support this hypothesis [3]. The findings of the current investigations suggest that for breath metabolites of presumed systemic origin, acetone and isoprene [3], measured on-line by non-standardized methods, $\mathrm{Cv}$ tends to be lower in comparison to orally released compounds, with the notable exception of ammonia. These results are, however, best explained by differences in the concentrations of these metabolites within the breath rather than their site of release (refer to the findings of Experiment I).

Ethanol, propanol, hydrogen cyanide, and acetic acid were found in higher concentrations within breath collected using a standardized sampling methodology (Mann Whitney $\mathrm{U}$ test; $P \leq 0.03$ ). With the possible exception of acetic acid, whose origin within the respiratory tree is yet to be confirmed, these metabolites are released in significant quantities from the oral cavity [3]. Findings might therefore suggest that the adoption of a standardized protocol, in particular the use of a restricted expiratory flow rate, leads to enrichment of the levels of orally generated breath metabolites. One possible explanation for this is that at lower expiratory flow rates the duration over which volatile metabolites are permitted to diffuse between the oral mucosa and gas phase is prolonged.

In contrast with the results for $\mathrm{Cv}$, the standard deviation of intra-subject differences in repeat breath samples, presented in Table 2 as precision, tended to be greater for standardized compared with nonstandardized breath samples. Further analysis revealed that differences were related to greater inter-subject variability in standardized experiments. Explanation of these results is likely to concern the influence of addi-
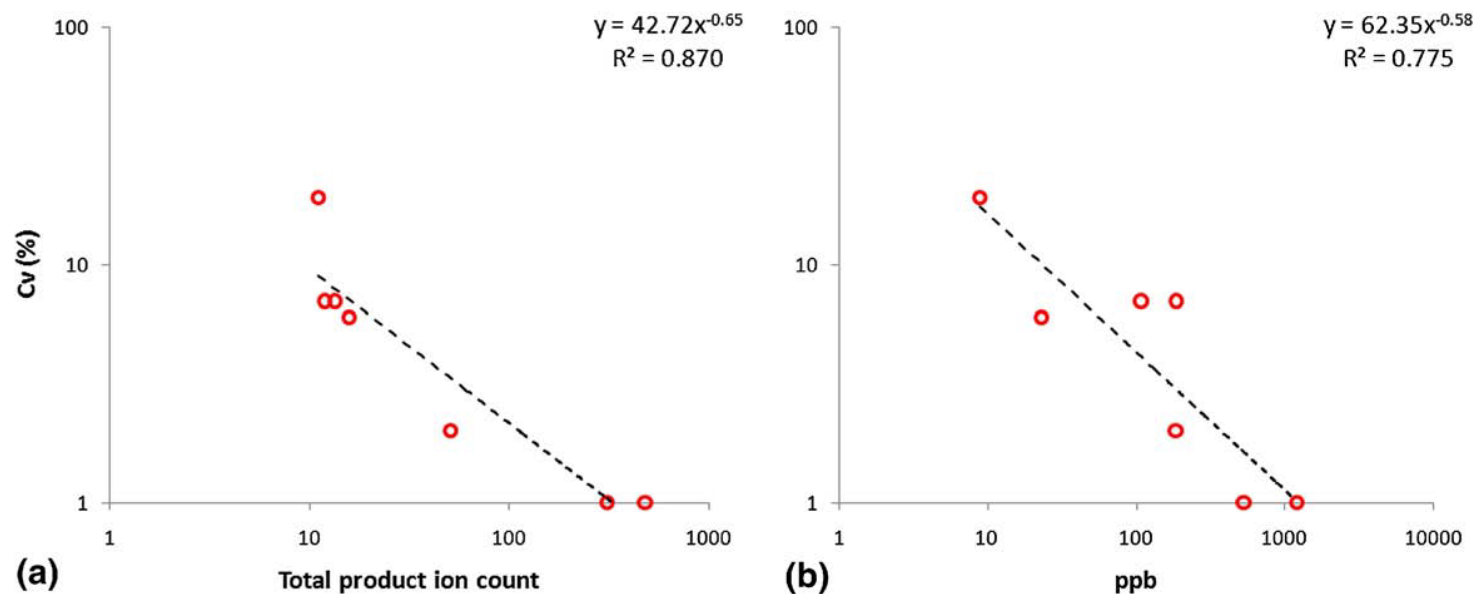

Figure 1. Scatter plots of the mean coefficient of variability, $\mathrm{Cv}(\%)$, as function of (a) total recorded product ion count and (b) ppb level, registered for the different breath metabolites listed in Table 1. 
Table 2. Repeatability of non-standardized [NS] and standardized [S] VOC analysis by SIFT-MS

\begin{tabular}{|c|c|c|c|c|c|c|c|c|}
\hline & \multicolumn{2}{|c|}{ Median (ppb) } & \multicolumn{2}{|c|}{ Precision $^{a}(p p b)$} & \multicolumn{2}{|c|}{$\mathrm{Rc}^{\mathrm{b}}$} & \multicolumn{2}{|c|}{$\mathrm{Cv}^{\mathrm{c}}$} \\
\hline & NS & $\mathrm{S}$ & NS & $\mathrm{S}$ & NS & $\mathrm{S}$ & NS & $\mathrm{S}$ \\
\hline Acetone & 598 & 507 & 73 & 78 & 0.43 & 0.90 & 0.02 & 0.02 \\
\hline Ethanol & 58 & 208 & 136 & 165 & 0.22 & 0.12 & 0.89 & 0.55 \\
\hline Propanol & 15 & 28 & 12 & 17 & 0.07 & 0.66 & 0.53 & 0.12 \\
\hline Hydrogen cyanide & 13 & 34 & 12 & 16 & 0.44 & 0.89 & 0.45 & 0.09 \\
\hline Isoprene & 281 & 251 & 118 & 150 & 0.73 & 0.90 & 0.25 & 0.09 \\
\hline Acetic acid & 77 & 145 & 64 & 80 & -0.17 & 0.08 & 0.90 & 0.40 \\
\hline Ammonia & 1007 & 1433 & 184 & 160 & 0.88 & 0.90 & 0.03 & 0.02 \\
\hline
\end{tabular}

aPrecision, standard deviation of the difference between repeated measures, calculated as: $2 / 3^{*}$ (breath $1-$ breath 3 ).

${ }^{\mathrm{b}} \mathrm{Rc}=$ Lin's concordance correlation coefficient.

${ }^{\mathrm{c}} \mathrm{Cv}=$ mean intra-individual coefficient of variability.

tional physiologic and environmental factors that require further investigation.

\section{Experiment III}

The repeatability of off-line mixed and alveolar breath sampling was assessed. Median values and repeatability statistics are presented in Table 3. Off-line measurement of acetone showed excellent repeatability in both mixed and alveolar samples. With the exception of acetic acid, alveolar breath sampling tended to improve repeatability of the analysis, while differences between Cv values for the two sampling approaches were less marked. The finding, in selected cases, of superior precision compared with results presented in Table 1 is attributed to the diminished influence of inter-subject variability.

The concentration of ethanol was observed to be higher within mixed compared with alveolar breath $(P=0.01)$ suggesting its release is predominantly from those regions of the respiratory tract that are not involved in gas exchange, including the oral cavity and larger airways. Whilst the opposite relationship was seen for isoprene, this failed to reach statistical significance $(P>0.05)$. It is therefore gratifying that current findings agree with the reports of previous authors who demonstrate ethanol and isoprene to be primarily of oral and systemic origin, respectively $[3,8]$.
In spite of the findings presented herein, there remains limited consensus as to the requirement for standardized practices within the field of exhaled breath analysis. It is unlikely that the adoption of guidelines similar to those proposed by the American Thoracic and European Respiratory Societies for the measurement of exhaled nitric oxide [9] will be justified for the majority of exhaled metabolites. Should standardization of the measurement of selected breath metabolites be thought necessary in the future, a greater understanding of their source of origin within the respiratory tree as well as knowledge of a defined association with both healthy and disease states is required. Further work is now needed to determine the influence of these factors as well as to more clearly define the effects of different sampling methodologies on the detection of exhaled metabolites. Allied with these investigations, continued refinement of the SIFT-MS technique, in particular improved sensitivity, will help support the analysis of volatile metabolites in the low ppb range and beyond.

A limitation of this study is that observations were made from only a relatively small number of healthy subjects. Whether findings are applicable to metabolites analyzed within the breath of patients with disease states remains unknown. Further work is needed to understand the relationship between pathologic status and measurement repeatability.

Table 3. Repeatability of SIFT-MS analysis of mixed [M] and alveolar [A] breath samples

\begin{tabular}{|c|c|c|c|c|c|c|c|c|}
\hline & \multicolumn{2}{|c|}{ Median (ppb) } & \multicolumn{2}{|c|}{ Precision $^{a}(p p b)$} & \multicolumn{2}{|c|}{$\mathrm{Rc}^{\mathrm{b}}$} & \multicolumn{2}{|c|}{$\mathrm{Cv}^{\mathrm{c}}$} \\
\hline & $M$ & $A$ & M & A & M & $A$ & $M$ & $A$ \\
\hline Acetone & 548 & 542 & 81 & 102 & 0.96 & 0.94 & 0.01 & 0.01 \\
\hline Ethanol & 203 & 108 & 63 & 62 & 0.41 & 0.59 & 0.05 & 0.06 \\
\hline Propanol & 22 & 17 & 15 & 5 & 0.60 & 0.71 & 0.08 & 0.05 \\
\hline Hydrogen cyanide & 10 & 10 & 10 & 5 & 0.18 & 0.68 & 0.23 & 0.17 \\
\hline Isoprene & 213 & 274 & 63 & 86 & 0.67 & 0.79 & 0.17 & 0.17 \\
\hline Acetic acid & 139 & 116 & 53 & 55 & 0.69 & 0.21 & 0.05 & 0.06 \\
\hline Ammonia & 1216 & 1301 & 827 & 791 & 0.27 & 0.67 & 0.04 & 0.04 \\
\hline
\end{tabular}

aprecision, standard deviation of the differences between repeated measurements.

${ }^{\mathrm{b}} \mathrm{Rc}=$ Lin's concordance correlation coefficient.

${ }^{\mathrm{c}} \mathrm{Cv}=$ mean intra-individual coefficient of variability. 


\section{Conclusions}

This study presents new evidence of the short-term repeatability of SIFT-MS analysis of prominent breath metabolites detected over a wide concentration range. For those metabolites investigated, this technique is both reliable and repeatable. Whilst sampling methodology does appear to have a role in influencing the level of observed repeatability and variability, metabolite concentration and physiologic variability remain the principal determinants of these parameters. Patterns of intra- and inter-individual variability in healthy subjects that are presented are intended to support interpretation of changes brought about by pathologic states, in turn helping to establish mass spectrometry-based methods within the clinical setting. Future work is now needed to determine the influence of other sampling methodologies as well as disease status on the day-today variation in exhaled metabolite levels.

\section{Acknowledgments}

The authors are grateful to Professor David Smith and Professor Patrik Španěl for their expert help and advice during the preparation of this manuscript. The authors acknowledge support for this study by a grant from the EU (framework 6), BAMOD, LSHC-CT-2005-019031.

\section{Appendix A Supplementary Material}

Supplementary material associated with this article may be found in the online version at doi:10.1016/ j.jasms.2010.02.008.

\section{References}

1. Smith, D.; Španěl, P. Selected Ion Flow Tube Mass Spectrometry (SIFTMS) for On-Line Trace Gas Analysis. Mass Spectrom. Rev. 2005, 24 661-700.

2. Beauchamp, J.; Herbig, J.; Gutmann, R.; Hansel, A. On the Use of Tedlar Bags for Breath-Gas Sampling and Analysis. J. Breath Res. 2008, 2, 046001.

3. Wang, T.; Pysanenko, A.; Dryahina, K.; Spaněl, P.; Smith, D. Analysis of Breath, Exhaled Via the Mouth and Nose, and the Air in the Oral Cavity. J. Breath Res. 2008, 2, 037013.

4. Pysanenko, A.; Španěl, P.; Smith, D. Analysis of the Isobaric Compounds Propanol, Acetic Acid, and Methyl Formate in Humid Air and Breath by Selected Ion Flow Tube Mass Spectrometry, SIFT-MS. Int. J. Mass Spectrom. 2009, 285, 42-48.

5. Warke, T. J.; Kamath, S.; Fitch, P. S.; Brown, V.; Shields, M. D.; Ennis, M. The Repeatability of Nonbronchoscopic Bronchoalveolar Lavage Differential Cell Counts. Eur. Respir. J. 2001, 18, 1009-1012.

6. Smith, D.; Turner, C.; Španěl, P. Volatile Metabolites in the Exhaled Breath of Healthy Volunteers: Their Levels and Distributions. J. Breath Res. 2007, 1, 014004

7. Španěl, P.; Dryahina, K.; Smith, D. A General Method for the Calculation of Absolute Trace Gas Concentrations in Air and Breath from Selected Ion Flow Tube Mass Spectrometry Data. Int. J. Mass Spectrom. 2006, 249/250, 230-239.

8. Schubert, J. K.; Spittler, K. H.; Braun, G.; Geiger, K.; Guttman, J. $\mathrm{CO}_{2}$-Controlled Sampling of Alveolar Gas in Mechanically Ventilated Patients. J. Appl. Physiol. 2001, 90, 486-492.

9. ATS/ERS Recommendations for Standardized Procedures for the OnLine and Off-Line Measurement of Exhaled Lower Respiratory Nitric Oxide and Nasal Nitric Oxide. Am. J. Respir. Crit. Care Med. 2005, 171, 912-930. 